Supplement of Biogeosciences Discuss., 12, 4365-4403, 2015

http://www.biogeosciences-discuss.net/12/4365/2015/

doi:10.5194/bgd-12-4365-2015-supplement

(C) Author(s) 2015. CC Attribution 3.0 License.

(c) (i)

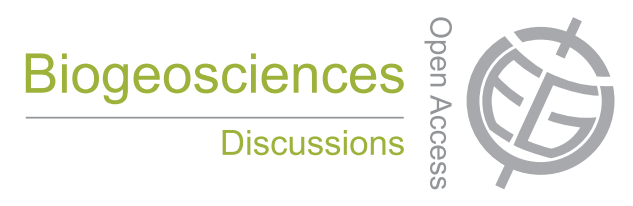

Supplement of

\title{
Major constrains of the pelagic food web efficiency in the Mediterranean Sea
}

\section{Zoccarato and S. Fonda Umani}

Correspondence to: L. Zoccarato (luca.zoccarato@phd.units.it) and S. Fonda Umani (s.fonda@units.it) 
Table 1: Chlorophyll a and biomasses values among all the dilution experiments carried out grouped per trophic conditions.

\begin{tabular}{l|l} 
Trophic conditions: & Oligotrophic
\end{tabular}

\begin{tabular}{|c|c|c|c|c|c|c|c|c|c|c|c|c|c|c|c|c|}
\hline & & Dec- & Mar- & & & Apr- & & & & & & & & & & \\
\hline Station: & VIERA & 99 & 04 & V3 & MS_03A & 04 & V4 & V1 & V2 & VA & V10 & O_37B & V6 & O_36 & CF_16 & V7 \\
\hline \multicolumn{17}{|l|}{$m g L^{-1}$} \\
\hline Chl a & 0.04 & 0.46 & 0.60 & 0.08 & 0.04 & 1.37 & 0.11 & 0.22 & 0.10 & 0.07 & 0.06 & 0.08 & 0.06 & 0.08 & 0.06 & 0.05 \\
\hline \multicolumn{17}{|l|}{ Biomass - $\mu g C L^{-1}$} \\
\hline MZP & 0.59 & 3.47 & 1.21 & 0.38 & 0.51 & 8.80 & 0.34 & 0.22 & 0.18 & 0.78 & 0.43 & 1.02 & 0.47 & 2.22 & 0.52 & 0.49 \\
\hline MPP & 0.04 & 0.13 & 0.12 & 1.68 & 0.23 & 1.01 & 2.53 & 2.73 & 2.75 & 3.24 & 3.30 & 0.13 & 3.76 & 0.34 & 0.28 & 5.89 \\
\hline HP & 4.87 & 21.02 & 5.84 & 10.85 & 4.71 & 5.13 & 11.70 & 13.15 & 9.84 & 11.70 & 6.14 & 5.80 & 7.49 & 6.32 & 4.49 & 5.89 \\
\hline AP & 0.42 & 0.57 & 0.94 & 0.44 & 2.60 & 1.86 & 0.82 & 0.70 & 0.88 & 0.62 & 0.64 & 3.91 & 0.49 & 4.43 & 4.71 & 0.45 \\
\hline
\end{tabular}

\begin{tabular}{|c|c|c|c|c|c|c|c|}
\hline Trophic conditions: & \multicolumn{7}{|c|}{ Mesotrophic } \\
\hline Station: & $\begin{array}{c}\text { Sep- } \\
03\end{array}$ & $\begin{array}{c}\text { Nov- } \\
00\end{array}$ & $\begin{array}{c}\text { Nov- } \\
01\end{array}$ & $\begin{array}{c}\text { Aug- } \\
05\end{array}$ & \multicolumn{2}{|r|}{ May- } & Aug-02 \\
\hline$m g L^{-1}$ & & & & & & & \\
\hline Chl a & 0.67 & 0.80 & 0.74 & 0.19 & 0.70 & 0.36 & 0.29 \\
\hline \multicolumn{8}{|l|}{ Biomass - $\mu g$ C $L^{-1}$} \\
\hline MZP & 7.21 & 3.48 & 5.40 & 1.85 & 12.04 & 2.35 & 6.05 \\
\hline MPP & 0.41 & 15.00 & 11.51 & 3.27 & 18.42 & 10.17 & 5.99 \\
\hline NP & 6.24 & 2.86 & 2.63 & 6.53 & 3.64 & 4.13 & 2.91 \\
\hline HP & 32.07 & 23.93 & 19.71 & 24.41 & 3.66 & 24.69 & 25.41 \\
\hline AP & 14.91 & 0.69 & 5.76 & 15.37 & 3.93 & 15.97 & 28.85 \\
\hline Trophic conditions: & \multicolumn{7}{|c|}{ Eutrophic } \\
\hline Station: & $\begin{array}{c}\text { Nov- } \\
98\end{array}$ & $\begin{array}{c}\text { May- } \\
99\end{array}$ & $\begin{array}{c}\text { Feb- } \\
01 \\
\end{array}$ & $\begin{array}{c}\text { May- } \\
00\end{array}$ & Aug-00 & $\begin{array}{c}\text { Feb- } \\
00 \\
\end{array}$ & Feb-99 \\
\hline$m g L^{-1}$ & & & & & & & \\
\hline Chl a & 1.64 & 1.15 & 3.39 & 0.61 & - & 2.90 & 5.94 \\
\hline \multicolumn{8}{|l|}{ Biomass - $\mu g C^{-1} L^{-1}$} \\
\hline MZP & 6.43 & 7.84 & 4.38 & 4.69 & 4.08 & 8.49 & 9.62 \\
\hline MPP & 124.95 & 145.25 & 157.17 & 201.17 & 255.09 & 300.68 & 1104.25 \\
\hline NP & 0.86 & 3.33 & 2.83 & 10.71 & 3.79 & 4.20 & 2.75 \\
\hline HP & 45.34 & 10.18 & 23.98 & 10.23 & 23.51 & 4.21 & 6.99 \\
\hline AP & 5.79 & 2.79 & 7.70 & 2.47 & 6.66 & 0.02 & 0.15 \\
\hline
\end{tabular}


Table 2a: Ingestion and potential production rates estimated among the considered dilution experiments carried out for the MZP and the HNF in oligotrophic conditions.

Trophic conditions:

Oligotrophic

\begin{tabular}{|c|c|c|c|c|c|c|c|c|c|c|c|c|c|c|c|c|c|}
\hline \multicolumn{2}{|c|}{$\begin{array}{cc}\text { Trophic conditions: } \\
\end{array}$} & \multicolumn{16}{|c|}{ Oligotrophic } \\
\hline & Station: & VIERA & Dec-99 & Mar-04 & V3 & MS_03A & Apr-04 & V4 & V1 & V2 & VA & V10 & O_37B & V6 & O_36 & CF_16 & V7 \\
\hline \multirow{10}{*}{ 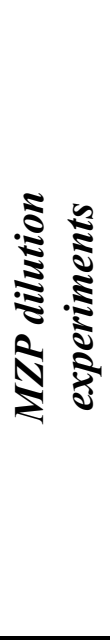 } & $\frac{\text { Ingestion rates }}{\mu g C L-1 d-1}$ & & & & & & & & & & & & & & & & \\
\hline & MPP & - & - & - & 1.04 & 0.09 & 0.71 & 0.03 & 0.05 & 0.06 & 0.75 & - & 0.02 & - & 0.04 & 0.01 & - \\
\hline & NP & - & - & 5.13 & - & 23.25 & 12.36 & 8.12 & 23.45 & - & 3.23 & 1.61 & 3.53 & 3.25 & - & - & 8.10 \\
\hline & HP & 10.18 & 7.81 & 2.72 & 3.36 & - & 1.80 & 23.84 & - & 23.73 & 4.41 & 13.19 & 5.70 & 8.64 & - & 1.97 & 9.42 \\
\hline & AP & - & 0.30 & 0.94 & 0.33 & 1.10 & 0.61 & - & - & 0.33 & 0.43 & - & 3.83 & - & 7.46 & 3.50 & - \\
\hline & $\frac{\text { Potential production rates }}{\mu g C L^{-1} d^{-1}}$ & & & & & & & & & & & & & & & & \\
\hline & МPP & - & - & - & 0.87 & 0.02 & 0.70 & 0.02 & 0.04 & 0.04 & 0.26 & - & 0.01 & - & 0.05 & 0.01 & - \\
\hline & NP & - & - & 3.15 & - & 7.47 & 7.37 & 4.65 & 15.26 & - & 0.62 & 1.15 & 1.59 & 3.60 & - & - & 8.89 \\
\hline & HP & 11.62 & 1.01 & 4.28 & 0.47 & - & 2.44 & 20.72 & - & 22.28 & 1.20 & 16.58 & 1.02 & 13.75 & - & - & 11.16 \\
\hline & AP & - & 0.29 & 0.85 & 0.26 & 0.08 & 0.68 & - & - & 0.14 & 0.07 & - & 4.07 & - & 7.86 & 2.95 & - \\
\hline \multirow{6}{*}{ 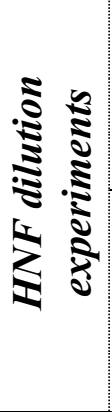 } & $\frac{\text { Ingestion rates }}{\mu g C L-1 d-1}$ & & & & & & & & & & & & & & & & \\
\hline & HP & 2.88 & 15.13 & 5.41 & 0.07 & - & 2.31 & 4.24 & 4.79 & 2.01 & 8.33 & 3.97 & 14.71 & 6.20 & 3.62 & 2.78 & 3.08 \\
\hline & AP & - & - & 0.81 & 0.09 & - & 2.03 & 1.06 & - & - & - & - & 7.55 & 0.14 & 4.39 & 0.42 & 0.36 \\
\hline & $\frac{\text { Potential production rates }}{\mu g C L^{-1} d^{-1}}$ & & & & & & & & & & & & & & & & \\
\hline & HP & 3.95 & 6.32 & 4.01 & 4.30 & - & 3.43 & 4.68 & 4.34 & 2.39 & 4.64 & 3.99 & 11.85 & 4.31 & 1.45 & 1.07 & 2.96 \\
\hline & AP & - & - & 0.72 & 0.03 & - & 2.13 & 0.53 & - & - & - & - & 6.95 & - & 2.57 & - & 0.36 \\
\hline
\end{tabular}


Table 2b: Ingestion and potential production rates estimated among the considered dilution experiments carried out for the MZP and the HNF in mesotrophic and eutrophic conditions.

\begin{tabular}{|c|c|c|c|c|c|c|c|c|c|c|c|c|c|c|c|c|c|c|c|}
\hline \multicolumn{2}{|r|}{ Trophic conditions: } & \multicolumn{11}{|c|}{ Mesotrophic } & \multicolumn{7}{|c|}{ Eutrophic } \\
\hline & Station: & $\begin{array}{c}\text { Sep- } \\
03 \\
\end{array}$ & $\begin{array}{c}\text { Nov- } \\
00\end{array}$ & $\begin{array}{c}\text { Nov- } \\
01\end{array}$ & $\begin{array}{c}\text { Aug- } \\
05 \\
\end{array}$ & $\begin{array}{c}\text { Mar- } \\
03 \\
\end{array}$ & $\begin{array}{c}\text { May- } \\
01 \\
\end{array}$ & $\begin{array}{c}\text { Aug- } \\
02 \\
\end{array}$ & $\begin{array}{l}\text { Aug- } \\
99 \\
\end{array}$ & $\begin{array}{c}\text { Feb- } \\
02 \\
\end{array}$ & $\begin{array}{c}\text { May- } \\
02 \\
\end{array}$ & $\begin{array}{c}\text { Aug- } \\
01\end{array}$ & $\begin{array}{c}\text { Nov- } \\
98 \\
\end{array}$ & $\begin{array}{l}\text { May- } \\
99 \\
\end{array}$ & $\begin{array}{c}\text { Feb- } \\
01\end{array}$ & $\begin{array}{c}\text { May- } \\
00\end{array}$ & $\begin{array}{l}\text { Aug- } \\
00\end{array}$ & $\begin{array}{c}\text { Feb- } \\
00\end{array}$ & $\begin{array}{l}\text { Feb- } \\
99\end{array}$ \\
\hline \multirow{10}{*}{ 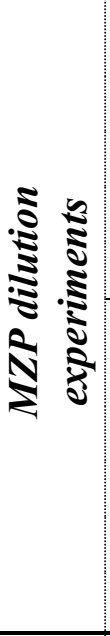 } & $\frac{\text { Ingestion rates }}{\mu g C L-1 d-1}$ & & & & & & & & & & & & & & & & & & \\
\hline & MPP & - & 9.95 & - & 2.30 & 10.34 & 3.27 & 4.53 & 1.46 & - & 23.49 & 19.54 & 50.97 & 70.02 & 84.23 & 155.19 & 176.21 & 113.90 & - \\
\hline & NP & 2.74 & - & - & 8.23 & 4.59 & 1.86 & 3.67 & - & - & 2.91 & 1.50 & 1.08 & 1.74 & 3.57 & 13.50 & - & 4.11 & 1.25 \\
\hline & HP & 44.04 & 40.12 & 11.34 & 29.82 & 4.59 & 32.16 & 37.77 & 39.43 & 35.47 & 27.64 & 53.53 & 9.38 & 10.47 & 9.83 & 23.71 & 66.90 & 2.25 & 5.16 \\
\hline & $\mathbf{A P}$ & 10.48 & 0.22 & - & 12.87 & - & 7.21 & 18.01 & 2.97 & 1.55 & 1.59 & 28.18 & 0.66 & 0.24 & 3.02 & 1.43 & 5.58 & 0.01 & 0.08 \\
\hline & $\frac{\text { Potential production rates }}{\mu g C L^{-1} d^{-1}}$ & & & & & & & & & & & & & & & & & & \\
\hline & MPP & - & 3.43 & - & 4.28 & 4.08 & 3.37 & 5.93 & 4.14 & - & 48.93 & 37.89 & 49.73 & 98.66 & 61.00 & 324.24 & 71.28 & 207.09 & - \\
\hline & NP & 1.13 & - & - & 4.91 & 2.74 & 0.67 & 2.19 & - & - & 2.14 & 5.44 & 0.65 & 0.66 & 2.13 & 8.05 & - & 1.94 & - \\
\hline & HP & 28.60 & 49.57 & 4.11 & 16.10 & 4.70 & 35.59 & 24.22 & 46.13 & 23.38 & 30.28 & 47.14 & 9.83 & 24.06 & 2.99 & 42.32 & 64.52 & 1.55 & 9.79 \\
\hline & $\mathbf{A P}$ & 6.71 & 0.23 & - & 8.38 & - & 2.27 & 7.06 & 2.75 & 0.85 & 0.83 & 13.13 & 0.83 & 0.36 & 2.86 & 0.93 & 1.12 & 0.02 & 0.04 \\
\hline \multirow{6}{*}{ 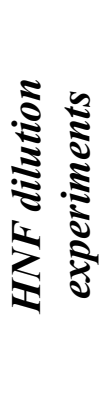 } & $\frac{\text { Ingestion rates }}{\mu g C L-1 d-1}$ & & & & & & & & & & & & & & & & & & \\
\hline & HP & 28.67 & 33.49 & 9.95 & 21.98 & 3.42 & - & 31.88 & 18.63 & 28.06 & 21.19 & 36.80 & 19.50 & 11.01 & - & 8.65 & 23.38 & - & 11.46 \\
\hline & AP & 8.30 & - & 1.45 & 6.69 & - & - & 10.75 & - & 1.78 & 1.19 & 37.66 & 0.38 & 0.61 & 0.38 & - & 0.42 & 0.01 & - \\
\hline & $\frac{\text { Potential production rates }}{\mu \mathrm{g} C \mathrm{~L}^{-1} d^{-1}}$ & & & & & & & & & & & & & & & & & & \\
\hline & HP & 13.48 & 41.45 & 1.76 & 11.03 & 2.29 & - & 22.46 & 26.42 & 18.27 & 23.48 & 23.46 & 13.87 & 21.72 & - & 17.74 & 27.58 & - & 13.55 \\
\hline & AP & 3.81 & - & 0.20 & 9.30 & - & - & 29.04 & - & 1.51 & 0.52 & 62.24 & - & 0.07 & 1.29 & - & 1.18 & 0.02 & - \\
\hline
\end{tabular}

